\title{
Correction to: Solve-RD: systematic pan-European data sharing and collaborative analysis to solve rare diseases
}

Birte Zurek (iD, Kornelia Ellwanger (D), Lisenka E. L. M. Vissers (iD, Rebecca Schüle, Matthis Synofzik (D), Ana Töpf, Richarda M. de Voer (iD, Steven Laurie (D), Leslie Matalonga, Christian Gilissen (D), Stephan Ossowski, Peter A. C. 't Hoen (ID, Antonio Vitobello (D), Julia M. Schulze-Hentrich, Olaf Riess, Han G. Brunner, Anthony J. Brookes (D), Ana Rath (D), Gisèle Bonne (D), Gulcin Gumus, Alain Verloes (iD, Nicoline Hoogerbrugge (D), Teresinha Evangelista, Tina Harmuth (D), Morris Swertz, Dylan Spalding (D), Alexander Hoischen, Sergi Beltran (iD, Holm Graessner (iD) Solve-RD consortium

(c) The Author(s), under exclusive licence to European Society of Human Genetics 2021

European Journal of Human Genetics (2021) 29:1459-1461; https://doi.org/10.1038/s41431-021-00936-4

Correction to: European Journal of Human Genetics https://doi.org/ 10.1038/s41431-021-00859-0

In the original publication of the article, consortium author list was missing in the article. The details is as below.

\section{Solve-RD consortium*}

Olaf Riess ${ }^{1,11}$, Tobias B. Haack ${ }^{1}$, Holm Graessner ${ }^{1,11}$, Birte Zurek ${ }^{1,11}$, Kornelia Ellwanger ${ }^{1,11}$, Stephan Ossowski ${ }^{1}$, German Demidov ${ }^{1}$ Marc Sturm ${ }^{1}$, Julia 'M. Schulze-Hentrich ${ }^{1}$, Rebecca Schüle ${ }^{4,5}$, Christoph Kessler ${ }^{4,5}$, Melanie Wayand ${ }^{4,5}$, Matthis Synofzik ${ }^{4,5}$, Carlo Wilke $^{4,5}$, Andreas Traschütz ${ }^{4,5}$, Ludger Schöls ${ }^{4,5}$, Holger Hengel ${ }^{4,5}$, Peter Heutink ${ }^{4,5}$, Han Brunner ${ }^{2,3,12}$, Hans Scheffer ${ }^{2,12}$, Nicoline' Hoogerbrugge $^{2,7}$, Alexander Hoischen ${ }^{2,7,20}$, Peter A.C. 't Hoen ${ }^{7,9}$ ' Lisenka E.L.M. Vissers ${ }^{2,3}$, Christian Gilissen ${ }^{2,7}$, Wouter Steyaert ${ }^{2,7}$ ', Karolis Sablauskas ${ }^{2}$, Richarda M. de Voer ${ }^{2,7}$, Erik-Jan Kamsteeg ${ }^{2}$, Bart van de Warrenburg ${ }^{3,23}$, Nienke van $\mathrm{Os}^{3,23}$, Iris te Paske ${ }^{2,7}$, Erik Janssen ${ }^{2,7}$, Elke de Boer ${ }^{2,3}$, Marloes Steehouwer ${ }^{2}$, Burcu Yaldiz ${ }^{2}$, Tjitske Kleefstra ${ }^{2,3}$, Anthony J. Brookes ${ }^{13}$, Colin Veal ${ }^{13}$, Spencer Gibson ${ }^{13}$, Marc Wadsley ${ }^{13}$, Mehdi Mehtarizadeh ${ }^{13}$, Umar Riaz ${ }^{13}$, Greg Warren ${ }^{13}$, Farid Yavari Dizjikan ${ }^{13}$, Thomas Shorter ${ }^{13}$, Ana' Töpf $^{6}$, Volker Straub ${ }^{6}$, Chiara Marini Bettolo ${ }^{6}$, Sabine Specht ${ }^{6}$, Jill Clayton-Smith ${ }^{24}$, Siddharth Banka ${ }^{24,25}$, Elizabeth Alexander ${ }^{24}$, Adam Jackson ${ }^{24}$, Laurence Faivre ${ }^{10,26,27,28,29}$, Christel Thau$\operatorname{vin}^{10,27,28,29}$, Antonio Vitobello ${ }^{10}$, Anne-Sophie DenomméPichon $^{10}$, Yannis Duffourd ${ }^{10,28}$, Emilie Tisserant ${ }^{10}$, Ange-Line Bruel $^{10}$, Christine Peyron ${ }^{30,31}$, Aurore Pélissier ${ }^{31}$, Sergi Beltran ${ }^{8,21}$, Ivo Glynne Gut ${ }^{21}$, Steven Laurie ${ }^{21}$, Davide Piscia ${ }^{21}$, Leslie' Matalonga ${ }^{21}$, Anastasios Papakonstantinou ${ }^{21}$, Gemma Bullich ${ }^{21}$, Alberto Corvo ${ }^{21}$, Carles Garcia ${ }^{21}$, Marcos Fernandez-Callejo ${ }^{21}$, Carles Hernández ${ }^{21}$, Daniel Picó ${ }^{21}$, Ida Paramonov ${ }^{21}$, Hanns Lochmüller $^{21}$, Gulcin Gumus ${ }^{32}$, Virginie Bros-Facer ${ }^{33}$, Ana Rath ${ }^{14}$, Marc Hanauer ${ }^{14}$, Annie Olry ${ }^{14}$, David Lagorce ${ }^{14}$, Svitlana Havrylenko ${ }^{14}$ Katia Izem ${ }^{14}$, Fanny Rigour ${ }^{14}$ Giovanni Steva$\operatorname{nin}^{34,35,36,37,38 \text { Alexandra Durr }{ }^{35,36,37,39} \text { Claire-Sophie }}$ Davoine ${ }^{35,36,37,38}$, Léna Guillot-Noel ${ }^{35,36,37,38}$, Anna Heinzmann $^{35,36,37,40}$, Giulia Coarelli ${ }^{35,36,37,40}$, Gisèle Bonne ${ }^{15}$, Teresinha Evangelista $^{15}$, Valérie Allamand ${ }^{15}$, Isabelle Nelson ${ }^{15}$, Rabah Ben Yaou $^{15,41,42}$, Corinne Metay ${ }^{15,43}$, Bruno Eymard ${ }^{15,41}$, Enzo Cohen ${ }^{15}$,
Antonio Atalaia ${ }^{15}$, Tanya Stojkovic ${ }^{15,41}$, Milan Macek Jr. ${ }^{44}$, Marek Turnovec ${ }^{44}$, Dana Thomasová ${ }^{44}$, Radka Pourová Kremliková ${ }^{44}$, Vera Franková ${ }^{44}$, Markéta Havlovicová ${ }^{44}$, Vlastimil Kremlik ${ }^{44}$, Helen Parkinson $^{19}$, Thomas Keane ${ }^{19}$, Dylan Spalding ${ }^{19}$, Alexander Senf ${ }^{19}$, Peter Robinson ${ }^{45}$, Daniel Danis ${ }^{45}$, Glenn Robert ${ }^{46}$, Alessia Costa ${ }^{46}{ }^{\prime}$ Christine Patch ${ }^{46,47}$, Mike Hanna ${ }^{48}$, Henry Houlden ${ }^{49}$, Mary Reilly ${ }^{48}{ }^{\prime}$ Jana Vandrovcova ${ }^{49}$, Francesco Muntoni ${ }^{50,51}$, Irina Zaharieva ${ }^{50}$, Anna Sarkozy ${ }^{50}$, Vincent Timmerman ${ }^{52,53}$, Jonathan Baets ${ }^{54,55,56}$, Liedewei Van de Vondel ${ }^{53,54}$, Danique Beijer ${ }^{53,54}$, Peter de Jonghe $e^{53,55}$, Vincenzo Nigro ${ }^{57,58}$, Sandro Banfi ${ }^{57,58}$ ' Annalaura Torella ${ }^{57}$, Francesco Musacchia ${ }^{57,58}$, Giulio Piluso ${ }^{57}$, Alessandra Ferlini $^{59}$, Rita Selvatici ${ }^{59}$, Rachele Rossi ${ }^{59}$, Marcella Neri ${ }^{59}$, Stefan Aretz $^{60,61}$, Isabel Spier ${ }^{60,61}$, Anna Katharina Sommer ${ }^{60}$,' Sophia Peters ${ }^{60}$, Carla Oliveira ${ }^{62,63,64}$, Jose Garcia Pelaez ${ }^{62,63}$, 'Ana Rita Matos $^{62,63}$ Celina São José ${ }^{62,63}$, Marta Ferreira ${ }^{62,63}$, Irene Gullo ${ }^{62,63,64}$, Susana Fernandes ${ }^{62,65}$ ', Luzia Garrido ${ }^{66}$, Pedro Ferreira ${ }^{62,63,67}$, Fátima Carneiro ${ }^{62,63,64}$, Morris A. Swertz ${ }^{18}$, Lennart Johansson ${ }^{18}$, Joeri K. van der Velde ${ }^{18}$, Gerben van der Vries ${ }^{18}$, Pieter B. Neerincx ${ }^{18}$, Dieuwke Roelofs-Prins ${ }^{18}$, Sebastian Köhler ${ }^{68}$, Alison Metcalfe ${ }^{46,69}$, Alain Verloes ${ }^{70,71}$, Séverine Drunat ${ }^{70,71}$, Caroline Rooryck ${ }^{72}$, Aurelien Trimouille ${ }^{73}$, Raffaele Castello ${ }^{58}$, Manuela Morleo ${ }^{58}$, Michele Pinelli ${ }^{58}$, Alessandra Varavallo ${ }^{58}$, Manuel Posada De la Paz ${ }^{74}$, Eva Bermejo Sánchez ${ }^{74}$, Estrella López Martín ${ }^{74}$, Beatriz Martínez Delgado ${ }^{74}$, F. Javier Alonso García de la Rosa ${ }^{74}$, Andrea Ciolf ${ }^{75}$, Bruno Dallapiccola ${ }^{75}$, Simone Pizzi ${ }^{75}$, Francesca Clementina Radio ${ }^{75}$, Marco Tartaglia ${ }^{75}$, Alessandra Renieri ${ }^{76,77,78}$, Elisa Benetti ${ }^{76}$, Peter Balicza ${ }^{79}$, Maria Judit Molnar ${ }^{79}$, Ales Maver ${ }^{80}$, Borut Peterlin ${ }^{80}$, Alexander Münchau ${ }^{81}$, Katja Lohmann ${ }^{81}$, Rebeccá Herzog ${ }^{81}$, Martje Pauly ${ }^{81}$, Alfons Macaya ${ }^{82}$, Anna Marcé-Grau ${ }^{82}$, Andres Nascimiento Osorio ${ }^{83}$, Daniel Natera de Benito ${ }^{83}$, Hanns Lochmüller ${ }^{84,85,86}$, Rachel Thompson ${ }^{85,86}$, Kiran Polavarapu ${ }^{84}$, David Beeson ${ }^{87}$,' Judith Cossins ${ }^{87}$, Pedro' M. Rodriguez Cruz ${ }^{87}$, Peter Hackman ${ }^{88}$, Mridul Johari ${ }^{88}$, Marco Savarese ${ }^{88}$, Bjarné Udd $^{88,89,90}$, Rita Horvath ${ }^{91}$, Gabriel Capella ${ }^{92}$, Laura Valle ${ }^{92}$, Elke Holinski-Feder ${ }^{93}$, Andreas Laner ${ }^{93}$, Verena Steinke-Lange ${ }^{93}$, Evelin Schröck ${ }^{94}$, Andreas Rump ${ }^{94,95}$

\footnotetext{
${ }^{23}$ Department of Neurology, Radboud University Medical Center, Nijmegen, The Netherlands;
} 
1460

${ }^{24}$ Division of Evolution and Genomic Sciences, School of Biological Sciences, Faculty of Biology, Medicine and Health, University of Manchester, Manchester, UK;

${ }^{25}$ Manchester Centre for Genomic Medicine, St Mary's Hospital, Manchester University Hospitals NHS Foundation Trust, Health Innovation Manchester, Manchester, UK;

${ }^{26}$ Dijon University Hospital, Genetics Department, Dijon, France;

${ }^{27}$ Dijon University Hospital, Centre of Reference for Rare Diseases, Development Disorders and Malformation Syndromes, Dijon, France;

${ }^{28}$ Dijon University Hospital, FHU- TRANSLAD, Dijon, France;

${ }^{29}$ Dijon University Hospital, GIMI Institute, Dijon, France;

${ }^{30}$ University of Burgundy-Franche Comté, Dijon Economics Laboratory, Dijon, France;

${ }^{31}$ University of Burgundy-Franche Comté, FHU-TRANSLAD, Dijon, France;

${ }^{32}$ EURORDIS-Rare Diseases Europe, Sant Antoni Maria Claret 167 08025, Barcelona, Spain;

${ }^{33}$ EURORDIS-Rare Diseases Europe, Plateforme Maladies Rares, Paris, France;

${ }^{34}$ Institut National de la Santé et de la Recherche Medicale (INSERM) U1127, Paris, France;

${ }^{35}$ Centre National de la Recherche Scientifique, Unité Mixte de Recherche (UMR) 7225, Paris, France;

${ }^{36}$ Unité Mixte de Recherche en Santé 1127, Université Pierre et Marie Curie (Paris 06), Sorbonne Universités, Paris, France;

${ }^{37}$ Institut du Cerveau -ICM, Paris, France;

${ }^{38}$ Ecole Pratique des Hautes Etudes, Paris Sciences et Lettres Research University, Paris, France;

${ }^{39}$ Centre de Référence de Neurogénétique, Hôpital de la PitiéSalpêtrière, Assistance Publique-Hôpitaux de Paris (AP-HP), Paris, France;

${ }^{40}$ Hôpital de la Pitié-Salpêtrière, Assistance Publique-Hôpitaux de Paris (AP-HP), Paris, France;

${ }^{41} \mathrm{AP}-\mathrm{HP}$, Centre de Référence de Pathologie Neuromusculaire Nord, Est, lle-de-France, Institut de Myologie, G.H. Pitié-Salpêtrière, Paris, France;

${ }^{42}$ Institut de Myologie, Equipe Bases de données, G.H. PitiéSalpêtrière, Paris, France;

${ }^{43} \mathrm{AP}-\mathrm{HP}$, Unité Fonctionnelle de Cardiogénétique et Myogénétique Moléculaire et Cellulaire, G.H. Pitié-Salpêtrière, Paris, France;

${ }^{44}$ Department of Biology and Medical Genetics, Charles University Prague-2nd Faculty of Medicine and University Hospital Motol, Prague, Czech Republic;

${ }^{45}$ Jackson Laboratory for Genomic Medicine, Farmington, CT, USA;
${ }^{46}$ Florence Nightingale Faculty of Nursing and Midwifery, King's College, London, UK;

${ }^{47}$ Genetic Counselling, Genomics England, Queen Mary University of London, Dawson Hall, London, UK;

${ }^{48}$ MRC Centre for Neuromuscular Diseases and National Hospital for Neurology and Neurosurgery, UCL Queen Square Institute of Neurology, London, UK;

${ }^{49}$ Department of Neuromuscular Diseases, UCL Queen Square Institute of Neurology, London, UK;

${ }^{50}$ Dubowitz Neuromuscular Centre, UCL Great Ormond Street Hospital, London, UK;

${ }^{51}$ NIHR Great Ormond Street Hospital Biomedical Research Centre, London, UK;

${ }^{52}$ Peripheral Neuropathy Research Group, Department of Biomedical Sciences, University of Antwerp, Antwerp, Belgium;

${ }^{53}$ Institute Born Bunge, Antwerp, Belgium;

${ }^{54}$ Peripheral Neuropathy Research Group, University of Antwerp, Antwerp, Belgium;

${ }^{55}$ Neuromuscular Reference Centre, Department of Neurology, Antwerp University Hospital, Antwerpen, Belgium;

${ }^{56}$ Laboratory of Neuromuscular Pathology, Institute Born-Bunge, University of Antwerp, Antwerpen, Belgium;

${ }^{57}$ Dipartimento di Medicina di Precisione, Università degli Studi della Campania "Luigi Vanvitelli,", Napoli, Italy;

${ }^{58}$ Telethon Institute of Genetics and Medicine, Pozzuoli, Italy;

${ }^{59}$ Unit of Medical Genetics, Department of Medical Sciences, University of Ferrara, Ferrara, Italy;

${ }^{60}$ Institute of Human Genetics, University of Bonn, Bonn, Germany;

${ }^{61}$ Center for Hereditary Tumor Syndromes, University Hospital Bonn, Bonn, Germany;

${ }^{62}$ i3S - Instituto de Investigação e Inovação em Saúde, Universidade do Porto, Porto, Portugal;

${ }^{63}$ IPATIMUP - Institute of Molecular Pathology and Immunology of the University of Porto, Porto, Portugal;

${ }^{64}$ Department of Pathology, Faculty of Medicine, University of Porto, Porto, Portugal;

${ }^{65}$ Department of Genetics, Faculty of Medicine, University of Porto, Porto, Portugal;

${ }^{66}$ CHUSJ, Centro Hospitalar e Universitário de São João, Porto, Portugal;

${ }^{67}$ Faculty of Sciences, University of Porto, Porto, Portugal;

${ }^{68}$ NeuroCure Cluster of Excellence, Charité Universitätsklinikum, Charitéplatz 1, Berlin, Germany; 
${ }^{69}$ College of Health, Well-being and Life-Sciences, Sheffield Hallam University, Sheffield, UK;

${ }^{70}$ Department of Genetics, Assistance Publique-Hôpitaux de Paris Université de Paris, Robert DEBRE University Hospital, 48 bd SERURIER, Paris, France;

${ }^{71}$ INSERM UMR 1141 "NeuroDiderot", Hôpital R DEBRE, Paris, France;

${ }^{72}$ Univ. Bordeaux, MRGM INSERM U1211, CHU de Bordeaux, Service de Génétique Médicale, Bordeaux, France;

${ }^{73}$ Laboratoire de Génétique Moléculaire, Service de Génétique Médicale, CHU Bordeaux - Hôpital Pellegrin, Place Amélie Raba Léon, Bordeaux Cedex, France;

${ }^{74}$ Institute of Rare Diseases Research, Spanish Undiagnosed Rare Diseases Cases Program (SpainUDP) \& Undiagnosed Diseases Network International (UDNI), Instituto de Salud Carlos III, Madrid, Spain;

${ }^{75}$ Genetics and Rare Diseases Research Division, Ospedale Pediatrico Bambino Gesù, IRCCS, Rome, Italy;

${ }^{76}$ Med Biotech Hub and Competence Center, Department of Medical Biotechnologies, University of Siena, Siena, Italy;

${ }^{77}$ Medical Genetics, University of Siena, Siena, Italy;

${ }^{78}$ Genetica Medica, Azienda Ospedaliero-Universitaria Senese, Siena, Italy;

${ }^{79}$ Institute of Genomic Medicine and Rare Diseases, Semmelweis University, Budapest, Hungary;

${ }^{80} \mathrm{Clinical}$ institute of genomic medicine, University medical centre Ljubljana, Ljubljana, Slovenia;

${ }^{81}$ Institute of Neurogenetics, University of Lübeck, Lübeck, Germany;
${ }^{82}$ Neurology Research Group, Vall d'Hebron Research Institute, Universitat Autònoma de Barcelona, Barcelona, Spain;

${ }^{83}$ Neuromuscular Disorders Unit, Department of Pediatric Neurology. Hospital Sant Joan de Déu, Barcelona, Spain;

${ }^{84}$ Department of Neuropediatrics and Muscle Disorders, Medical Center, Faculty of Medicine, University of Freiburg, Freiburg, Germany;

${ }^{85}$ Centro Nacional de Análisis Genómico (CNAG- CRG), Center for Genomic Regulation, Barcelona Institute of Science and Technology (BIST), Barcelona, Spain;

${ }^{86}$ Children's Hospital of Eastern Ontario Research Institute, University of Ottawa, Ottawa, ON, Canada;

${ }^{87}$ Nuffield Department of Clinical Neurosciences, University of Oxford, Oxford, UK;

${ }^{88}$ Folkhälsan Research Centre and Medicum, University of Helsinki, Helsinki, Finland;

${ }^{89}$ Tampere Neuromuscular Center, Tampere, Finland;

${ }^{90}$ Vasa Central Hospital, Vaasa, Finland;

${ }^{91}$ Department of Clinical Neurosciences, University of Cambridge, Cambridge, UK;

${ }^{92}$ Bellvitge Biomedical Research Institute (IDIBELL), Barcelona, Spain;

${ }^{93}$ Medical Genetics Center (MGZ), Munich, Germany;

${ }^{94}$ Institute for Clinical Genetics, Faculty of Medicine Carl Gustav Carus, Technical University Dresden, Dresden, Germany;

${ }^{95}$ Center for Personalized Oncology, University Hospital Carl Gustav Carus, Technical University Dresden, Dresden, Germany 